\title{
DUAL LIE ELEMENTS AND A DERIVATION FOR THE COFREE COASSOCIATIVE COALGEBRA
}

\author{
GARY GRIFFING
}

(Communicated by Lance W. Small)

\begin{abstract}
We construct a derivation $D$ in the Hopf algebra $T c V$, the cofree coassociative coalgebra on a vector space $V$. We then define the subspace of $T c V$ consisting of dual Lie elements, which is analogous to the subspace of the Hopf algebra $T V$, the free associative algebra on $V$, consisting of Lie elements. Thereafter, we formulate a dual Dynkin-Specht-Wever theorem. Using our map $D$, we then give very short proofs of both the dual DynkinSpecht-Wever and dual Friedrichs' theorems, each of which characterizes the space of dual Lie elements in $T c V$ at characteristic 0 .
\end{abstract}

\section{INTRODUCTION AND BACKGROUND}

Let $V$ be a vector space over a field $F$, and let $T c V$ denote the cofree coassociative coalgebra on $V$, with comultiplication $\Delta$ and canonical linear projection $\pi: T c V \rightarrow V$. We recall that $T c V$ is a Hopf algebra whose elements may be expressed [B-L, p. 17; B, p. 278] as certain formal infinite sums $f=$ $\sum_{0 \leq n} f^{n}$, where $f^{n} \in V^{\otimes n}$ (the degree $n$ component of both $T V$ and $s h V$, the tensor and shuffle algebras on $V$, respectively). We also let $\widehat{s h V}$ denote the completion of $s h V$, that is, the vector space of all formal infinite sums $f=\sum_{0 \leq n} f^{n}\left(f^{n} \in V^{\otimes n}\right)$ equipped with extended shuffle multiplication. Thus, if we denote shuffle multiplication in $\operatorname{sh} V$ by $\bullet$, then $\widehat{s h V}$ has multiplication (also denoted $\bullet$ ) defined by

$$
f \bullet g=\sum_{0 \leq n} \sum_{i+j=n} f^{i} \bullet g^{j} .
$$

As an algebra, $T c V \subseteq \widehat{s h V}$ is a subalgebra [B, p. 284].

We first construct a specific derivation $D: \overline{s h V} \rightarrow \overline{s h V}$, under which the subalgebra $T c V$ is invariant (Proposition 1). The map $D$ will have the following effect on $\widehat{s h V}$ and hence on $T c V$ : For $f \in \widehat{s h V}, D f=\sum_{0 \leq n} n f^{n}$; thus, $D$ can be considered a degree derivation. Next, we construct a useful map $\rho: \widehat{s h V} \rightarrow \widehat{s h V}$ and show (Proposition 4) that the subalgebra $T c V$ is invariant under $\rho$. The map $\rho$ gets used in an essential way in the proofs of our main

Received by the editors February 3, 1993 and, in revised form, April 15, 1994.

1991 Mathematics Subject Classification. Primary 17B99; Secondary 16A24. 
results, Theorem 6 and Theorem 8 , of section 3. We comment that Lemma 3 shows that our map $\rho_{n}$, which appears in the construction of the map $\rho$, turns out to be equivalent to the map denoted by $\sigma$ in [R, p. 217]. (The algebra $\mathscr{U}$ of $[R$, p. 210] is isomorphic as an algebra to $s h V$ (over a commutative base ring), via $\alpha\left(i_{1} \cdots i_{n}\right) \mapsto v_{i_{1}} \otimes \cdots \otimes v_{i_{n}}$.) Moreover, the respective contexts of these maps appear to be dual to one another.

Recall that the space of Lie elements is defined as the subalgebra of $\mathscr{L}(T V)$ generated by $l(V)$, where $\mathscr{L}(T V)$ is the Lie algebra associated to the associative algebra $T V$ and $l: V \rightarrow T V$ is the canonical linear injection [J, p. 168]. (We mention that $\mathscr{L}(T V)$ is also frequently denoted by $T V^{-}$.) In section 3 , we define the space of dual Lie elements of $T c V$ by dual analogy with the space of Lie elements of $T V$. Next, for $F$ of characteristic 0 , we formulate a dual version of the classical Dynkin-Specht-Wever theorem (Theorem 6) using the map $D$. In Theorem 8 we apply $D$ and $\rho$ to give extremely short proofs of both the dual Dynkin-Specht-Wever and the dual Friedrichs' theorems. (An earlier and longer proof of the dual Friedrichs' theorem is given in [G, Theorem 11]). These theorems characterize the dual Lie elements (of $T c V$ ) in a manner analogous to the characterization of the Lie elements (of $T V$ ) by the well-known Friedrichs' and Dynkin-Specht-Wever theorems, as given in [R, $p$. 214].

Since the Friedrichs' and Dynkin-Specht-Wever theorems can be used to prove the Campbell-Baker-Hausdorff formula [J, p. 172], one can wonder about a possible "dual C-B-H formula".

We will now proceed to recall the coalgebra structure of $T c V$. Given $f \in$ $\widehat{s h V}$, we say that a finite subset

$$
\left\{g_{s}, h_{s} \in \widehat{s h V}: s \in S\right\}
$$

represents (or forms a set of representing elements for) $f$ if whenever $0 \leq n$, $i+j=n$, we have that $f^{n}=\sum_{s \in S} g_{s}^{i} \otimes h_{s}^{j}$, as an element $V^{\otimes i} \otimes V^{\otimes j} \subseteq V^{\otimes i+j}$. The coalgebra $T c V$ consists of those $f \in \widehat{s h V}$ for which there exists a corresponding finite subset of representing elements. Such elements $f$ are called representative; one also shows that the representing elements are themselves representative [B-L, p. 16]. The coalgebra structure maps are now easy to describe. Let $f=\sum_{0 \leq n} f^{n} \in T c V$; then the comultiplication $\Delta: T c V \rightarrow T c V \otimes T c V$, the counit $\varepsilon: T c V \rightarrow F$, and canonical projection $\pi: T c V \rightarrow V$ are given respectively by

$$
\Delta(f)=\sum_{s \in S} g_{s} \otimes h_{s}, \quad \varepsilon(f)=f^{0}, \quad \text { and } \quad \pi(f)=f^{1} .
$$

Thus, for $f \in T c V$, we have (using the notation in [S]) $\Delta(f)=\sum_{(f)} f_{(1)} \otimes f_{(2)}$. By [B, p. 277], for $1 \leq n$ we have $f^{n}=\left(\otimes^{n} \pi\right) \Delta_{n-1}(f)$, that is,

$$
f^{n}=\sum_{(f)} f_{(1)}^{1} \otimes \cdots \otimes f_{(n)}^{1} .
$$

We now recall from [B-L, p. 20] a useful criterion for determining which $f \in$ $\widehat{s h V}$ are representative. Let $\left\{v_{k}: k \in K\right\}$ be a basis of $V$ and $\lambda_{j} \in V^{*}$ with 
$\lambda_{j}\left(v_{k}\right)=\delta_{j k}$, for $j, k \in K$. Define a left truncated translation operator

$$
L_{j}: \widehat{s h V} \rightarrow \widehat{s h V}, \quad \sum_{0 \leq n} f^{n} \mapsto \sum_{0 \leq n}\left(\lambda_{j} \otimes\left(\bigotimes^{n} 1\right)\right) f^{n+1}, \quad \text { where } 1=\text { Id. }
$$

There is an analogous right truncated translation operator $R_{j}$, the effect of which is to replace $\lambda_{j} \otimes\left(\otimes^{n} 1\right)$ by $\left(\otimes^{n} 1\right) \otimes \lambda_{j}$ in the above formula. Using $L_{j}$ rather than $R_{j}$ in Corollary 6 of [B-L], we have the following characterization, corresponding to the usual characterization of a representative function on a semigroup $G$ as an element of $\operatorname{Map}(G, F)$ whose space of left (or right) translates is finite dimensional.

Lemma 0. Let $f \in \widehat{s h V}$ be given. Then $f \in T c V$ if and only if

$$
\operatorname{span}\left\{L_{j_{1}} \cdots L_{j_{m}} f: j_{i} \in K, i=1, \ldots, m, 0 \leq m\right\}
$$

is finite dimensional.

Let $L f$ denote the above span, and define $R f$ similarly. Then by Corollary 5 of [B-L] applied to $T c V\left(=F[x]_{V}^{0}\right)$, we have that the finite-dimensional subcoalgebra generated by $f \in T c V$ is $L R f=R L f$.

\section{The DeRIVATION $D$ AND THE MAP $\rho$}

We will now introduce and examine our derivation $D$ and a closely related map $\rho$. Thereafter, in Proposition 1 we will show that $T c V$ is invariant under $D$, and in Proposition 4 that $T c V$ is invariant under $\rho$; thus, we will show that if $f \in T c V$, then $D f \in T c V$ and $\rho f \in T c V$.

We mention that one can show that $D$ is the convolution product $\rho * 1$ of $\rho$ and 1, the identity, in $\operatorname{Hom}(T c V, T c V)$. That is, by expanding and using (2), one has $D=(\bullet)(\rho \otimes 1) \Delta$. This latter fact is not necessary for this work, but is interesting nevertheless.

Define a linear map

$$
D: \widehat{s h V} \rightarrow \widehat{s h V}, \quad \sum_{0 \leq n} f^{n} \mapsto \sum_{0 \leq n} n f^{n} .
$$

Proposition 1. The linear map $D$ is a derivation of the algebra $\widehat{s h V}$, and the subalgebra $T c V$ is invariant under $D$. Moreover, $D$ is a coderivation $(\Delta D=$ $(1 \otimes D+D \otimes 1) \Delta)$ of $T c V$.

Proof. We first show the derivation property of $D$. Let $f=\sum_{0 \leq n} f^{n}$ and $g=\sum_{0 \leq n} g^{n}$. We find that $D(f \bullet g)=\sum_{0 \leq n} n \sum_{i+j=n} f^{i} \bullet g^{j}$, while on the other hand,

$$
\begin{aligned}
(D f) \bullet g+f \bullet(D g) & =\sum_{0 \leq i} i f^{i} \bullet \sum_{0 \leq j} g^{j}+\sum_{0 \leq i} f^{i} \cdot \sum_{0 \leq j} j g^{j} \\
& =\sum_{0 \leq n} \sum_{i+j=n} i f^{i} \bullet g^{j}+\sum_{0 \leq n} \sum_{i+j=n} j f^{i} \bullet g^{j} \\
& =\sum_{0 \leq n} \sum_{i+j=n}(i+j) f^{i} \bullet g^{j}=\sum_{0 \leq n} n \sum_{i+j=n} f^{i} \bullet g^{j} .
\end{aligned}
$$

We now show that $T c V$ is invariant under $D$. Let $f \in T c V$ and $\Delta(f)=$ $\sum_{s \in S} g_{s} \otimes h_{s}$ so that $f^{n}=\sum_{s \in S} g_{s}^{i} \otimes h_{s}^{j}$, whenever $i+j=n$. Therefore, for 
each $0 \leq n$ and $i+j=n$,

$$
\begin{aligned}
(D f)^{n} & =n f^{n}=\sum_{s \in S}\left(D g_{s}^{i} \otimes h_{s}^{j}+g_{s}^{i} \otimes D h_{s}^{j}\right) \\
& =\sum_{s \in S}\left(\left(D g_{s}\right)^{i} \otimes h_{s}^{j}+g_{s}^{i} \otimes\left(D h_{s}\right)^{j}\right) .
\end{aligned}
$$

This shows that $\left\{g_{s}, D g_{s}, h_{s}, D h_{s}: s \in S\right\}$ forms a set of representing elements for $D f$; therefore, $D f \in T c V$ and $\Delta(D f)=\sum_{s \in S}\left(D g_{s} \otimes h_{s}+g_{s} \otimes D h_{s}\right)$.

Recall that $\operatorname{sh} V$ (=TV as a vector space) is the irreducible component of 1 in $T c V$ and can be identified as a Hopf algebra with the sub Hopf algebra of $T c V$ consisting of those formal sums which are finitely non-zero [B, p. 282]. For each $0 \leq n$, we inductively define a linear map $\rho_{n}: \operatorname{sh} V \rightarrow \operatorname{sh} V$, where $\tau=$ twist map and $\Delta=\Delta_{s h V}$. Set $\rho_{0}=0, \rho_{1}=1$, and for $2 \leq n$

$$
\rho_{n}=\left(\bigotimes^{n-2} 1 \otimes(1-\tau) \Delta\right) \rho_{n-1}
$$

Thus, we have a linear map

$$
\rho: \widehat{s h V} \rightarrow \widehat{s h V}, \quad \sum_{0 \leq n} f^{n} \mapsto \sum_{0 \leq n} \rho_{n} f^{n} .
$$

We will now show that $\rho$ maps $T c V$ to $T c V$. In order to do this, we need the following two technical lemmas. For $1 \leq j$ and $v_{1}, \ldots, v_{j} \in V$, we will write $v_{1} \cdots v_{j}$ to mean the homogeneous tensor $v_{1} \otimes \cdots \otimes v_{j}$.

Lemma 2. If $2 \leq n, 1 \leq m \leq n-1$, and $v_{1} \cdots v_{m} \in V^{\otimes m}$, then

$$
\rho_{n}\left(v_{1} \cdots v_{m}\right)=0 \text {. }
$$

Proof. It suffices to assume that for any $n, m=n-1$. Now use induction on $n$, the base step being clear since

$$
(1-\tau) \Delta(v)=(1-\tau)(1 \otimes v+v \otimes 1)=0,
$$

for all $v \in V$.

We remark that if $\rho_{n}$ were defined on the left (rather than the right) as $\rho_{n}=\left((1-\tau) \Delta \otimes\left(\otimes^{n-2} 1\right)\right) \rho_{n-1}$, the following lemma would show that $\rho_{n}$ is identical to the map denoted by $\sigma$ in [R, p. 217] (see comment in section 1).

Lemma 3. If $2 \leq n$ and $v_{1} \cdots v_{n} \in V^{\otimes n}$, then

$$
\rho_{n}\left(v_{1} \cdots v_{n}\right)=v_{1} \otimes \rho_{n-1}\left(v_{2} \cdots v_{n}\right)-v_{n} \otimes \rho_{n-1}\left(v_{1} \cdots v_{n-1}\right)
$$

Proof. Using induction on $n$, we have that $\rho_{n}=\left(1 \otimes \rho_{n-1}\right)(1-\tau) \Delta$. Lemma 2 now implies

$$
\begin{aligned}
\rho_{n}\left(v_{1} \cdots v_{n}\right)=\left(1 \otimes \rho_{n-1}\right)\left(v_{1} \otimes v_{2} \cdots v_{n}-v_{2} \cdots v_{n} \otimes v_{1}\right. \\
\left.+v_{1} \cdots v_{n-1} \otimes v_{n}-v_{n} \otimes v_{1} \cdots v_{n-1}+\sum L t \otimes L t^{\prime}\right) \\
=v_{1} \otimes \rho_{n-1}\left(v_{2} \cdots v_{n}\right)-v_{n} \otimes \rho_{n-1}\left(v_{1} \cdots v_{n-1}\right)
\end{aligned}
$$

where $L t$ and $L t^{\prime}$ denote elements of degree less than $n-1$. 
Later in Proposition 7, we will require the following identity, which is given in Lemma 1.2 of [R, p. 212] (see comment in section 1). This identity can be easily proved by induction. For $1 \leq n$ and $v_{1}, \ldots, v_{n} \in V$,

$$
n\left(v_{1} \cdots v_{n}\right)=\sum_{i=1}^{n} \rho_{i}\left(v_{1} \cdots v_{i}\right) \bullet\left(v_{i+1} \cdots v_{n}\right) .
$$

For the following proposition, we use the basis of $V$ and notation chosen in the previous section; thus, let $\lambda_{j} \in V^{*}(j \in K)$ be defined as above.

Proposition 4. If $f \in T c V$, then $\rho f \in T c V$.

Proof. Let $f \in T c V$. Using the notation of section 1, we will show that $L(\rho f)$ is finite dimensional. Lemma 0 will then imply that $\rho f \in T c V$. Suppose that $\Delta(f)=\sum_{s \in S} g_{s} \otimes h_{s}$. By the proof of Theorem 3 in [B-L], we may take the $g_{s}$ and the $h_{s}(s \in S)$ as spanning sets for $R f$ and $L f$ respectively. In order to invoke Lemma 0 we consider the following calculation, where $0 \leq n$. By (1) and Lemma 3, we find

$$
\begin{aligned}
&\left(\lambda_{j} \otimes\right.\left.\left(\otimes^{n} 1\right)\right) \rho_{n+1} f^{n+1}=\left(\lambda_{j} \otimes\left(\bigotimes^{n} 1\right)\right) \rho_{n+1} \sum_{(f)} f_{(1)}^{1} \otimes \cdots \otimes f_{(n+1)}^{1} \\
&=\left(\lambda_{j} \otimes\left(\bigotimes^{n} 1\right)\right) \sum_{(f)}\left\{f_{(1)}^{1} \otimes \rho_{n}\left(f_{(2)}^{1} \otimes \cdots \otimes f_{(n+1)}^{1}\right)\right. \\
&\left.\quad-f_{(n+1)}^{1} \otimes \rho_{n}\left(f_{(1)}^{1} \otimes \cdots \otimes f_{(n)}^{1}\right)\right\} \\
& \quad=\sum_{s \in S}\left\{\lambda_{j}\left(g_{s}^{1}\right) \rho_{n}\left(h_{s}^{n}\right)-\lambda_{j}\left(h_{s}^{1}\right) \rho_{n}\left(g_{s}^{n}\right)\right\} .
\end{aligned}
$$

Therefore, summing on $n$ gives

$$
\begin{aligned}
L_{j}(\rho f) & =\sum_{0 \leq n}\left(\lambda_{j} \otimes\left(\otimes^{n} 1\right)\right) \rho_{n+1} f^{n+1} \\
& =\sum_{0 \leq n} \sum_{s \in S}\left\{\lambda_{j}\left(g_{s}^{1}\right) \rho_{n}\left(h_{s}^{n}\right)-\lambda_{j}\left(h_{s}^{1} j\right) \rho_{n}\left(g_{s}^{n}\right)\right\} \\
& =\sum_{s \in S}\left\{\alpha_{s} \sum_{0 \leq n} \rho_{n}\left(h_{s}^{n}\right)-\beta_{s} \sum_{0 \leq n} \rho_{n}\left(g_{s}^{n}\right)\right\} \\
& =\sum_{s \in S}\left(\alpha_{s} \rho\left(h_{s}\right)-\beta_{s} \rho\left(g_{s}\right)\right),
\end{aligned}
$$

where $\alpha_{s}$ and $\beta_{s}$ are the scalars $\lambda_{j}\left(g_{s}^{1}\right)$ and $\lambda_{j}\left(h_{s}^{1}\right)$ respectively. We now have that

$$
\left\{\rho\left(h_{s}\right), \rho\left(g_{s}\right)\right\}_{s \in S}
$$

is a finite spaning set for $L_{j}(\rho f)$. We have just shown that for any $j \in K$, $L_{j}(\rho f) \subseteq \operatorname{span}\{\rho(R f), \rho(L f)\}$. Hence, by Corollary 5 of [B-L], $L(\rho f) \subseteq$ $\operatorname{span}\{\rho(L R f)\}$, which is finite dimensional. By Lemma 0 we have that $\rho f \in$ $T c V$; that is, $\rho$ maps $T c V$ to $T c V$.

The maps $D$ and $\rho$ were each defined with domain and codomain $\widehat{s h V}$. In the previous two propositions it was shown that $D$ and $\rho$, each with their domains restricted to $T c V$, have codomains $T c V$, respectively. With this 
in mind and for ease in notation, we will continue to denote by $D$ and $\rho$ the corresponding maps, each of which now have their domain and codomain restricted to $T c V$.

\section{Applications to duAl Lie elements}

In this section, $F$ has characteristic 0 .

As was mentioned in the introduction, if $l: V \rightarrow T V$ is the canonical linear injection, then the space of Lie elements is the smallest subalgebra of $\mathscr{L}(T V)$ containing $l(V)$. That is, the space of Lie elements equals the subalgebra of $\mathscr{L}(T V)$ generated by $l(V)$. This subalgebra can also be described as the space spanned by all iterated brackets of elements of $V$. To define dual Lie elements, we must first recall the notation of a Lie coalgebra. A Lie coalgebra $(K, \delta)$ is a vector space $K$ and a linear map $\delta: K \rightarrow K \otimes K$, satisfying identities which are arrows-reversed versions of the defining identities for a Lie algebra $[G, p$. $447 ; M$, p. 4]. The cofree Lie coalgebra (on $V$ ), that is, the cofree object in the category of Lie coalgebras, is denoted by $L c V$ and satisfies the usual universal mapping property. (This u.m.p. is obtained by reversing arrows in the u.m.p. satisfied by the free Lie algebra on $V$.) If $(C, \Delta)$ is a coassociative coalgebra, $\mathscr{L}_{c}(C)$ will denote the associated Lie coalgebra whose underlying vector space is $C$ and whose comultiplication is $(1-\tau) \Delta$. A covered Lie coalgebra is a vector space $K$ together with a linear map $\delta: K \rightarrow K \otimes K$ for which there exists a coassociative, counital, coalgebra $(C, \Delta, \varepsilon)$ and a surjective map $\omega: \mathscr{L}_{c}(C) \rightarrow K$ such that $(\omega \otimes \omega)(1-\tau) \Delta=\delta \omega$. All covered Lie coalgebras are Lie coalgebras, but not all Lie coalgebras are covered [M, p. 9]. Thus, the category of covered Lie coalgebras is strictly smaller than the category of Lie coalgebras. In fact, in the proposition in [M, p. 41] it is shown that a Lie coalgebra is covered if and only if it is locally finite, that is, if and only if each element lies in a finite-dimensional Lie subcoalgebra. Moreover, it is precisely the covered Lie coalgebras in which the dual P-B-W theorem holds [A, B, M1]. Given a Lie coalgebra $K$, a subspace $I \subseteq K$ is a Lie coideal if $\delta(I) \subseteq K \otimes I+I \otimes K$. (There exists a well-known duality between the coideals of a coalgebra $C$ and the subalgebras of the dual algebra $C^{*}$; thus, quotient coalgebras of $C$ correspond to subalgebras of $C^{*}$; see [S, p. 19] for coassociative coalgebras, and essentially the same argument works for Lie coalgebras.) The category of covered Lie coalgebras also has a cofree object on $V$. This cofree object is denoted by $\mathscr{L}_{c}(T c V) / J$, where $J$ is the largest Lie coideal (sum of all such) of $\mathscr{L}_{c}(T c V)$ contained in $\operatorname{ker} \pi$. Thus, we say that $J$ is the Lie coideal cogenerated by ker $\pi$. The quotient $\mathscr{L}_{c}(T c V) / J$ corresponds to the subalgebra of $\mathscr{L}(T V)$ generated by $l(V)$. Thus, the Lie coideal $J$ of $\mathscr{L}_{c}(T c V)$ corresponds to the subalgebra of $\mathscr{L}(T V)$ consisting of Lie elements.

Therefore, elements dual to the Lie elements would be elements of $J$. This is the motivation for the following definition.

Definition. A dual Lie element is any element of $J$. Thus, $f \in T c V$ is a dual Lie element if $f$ is a member of the largest Lie coideal contained in the kernel of the canonical linear projection $\pi: T c V \rightarrow V$, that is, if $f$ is a member of the Lie coideal cogenerated by $\operatorname{ker} \pi$.

However, as we will see in Theorem 8 in contrast to the space of Lie elements, the space of dual Lie elements contains the (Lie coideal) base field $F$. 
Recall that for any counital coalgebra $C$, we write $\bar{C}=\operatorname{ker} \varepsilon$; in particular, $\overline{T c V}$ is contained in all formal infinite sums with degree 0 -component equaling 0 , and $\overline{T V}=\bigoplus_{1 \leq n} V^{\otimes n}$. We also have the following result, which is stated in [M, p. 8] and proved in both [B, Lemma 5.1] in [M2, p. 138].

Lemma 5. If $B$ is any bialgebra, then $(1-\tau) \Delta\left(\bar{B}^{2}\right) \subseteq \bar{B}^{2} \otimes B+B \otimes \bar{B}^{2}$. That is, regarding $B$ as a coalgebra, $\bar{B}^{2}$ is a Lie coideal in the associated Lie coalgebra $\mathscr{L}_{c}(B)$.

In [G, p. 458] we gave the dual Friedrichs' theorem characterizing dual Lie elements. Before we recall this theorem, we will recall two versions of Friedrichs' theorem, each of which characterizes Lie elements. The second version will lend itself to dualization. Let $\theta: T V \rightarrow T V \otimes T V$ be the linear map extending $v \mapsto 1 \otimes v+v \otimes 1, v \in V$. Also for each $1 \leq n$, let $\left\{u_{i}: i \in B_{n}\right\}$ be a basis of $V^{\otimes n}$, constructed from a basis of $V$, and $\left\{u_{i}: i \in \bigcup B_{n}\right\}$ be the resulting basis of $\overline{T V}$. Let $\langle\rangle:, \overline{T V} \times \overline{T V} \rightarrow F$ be the (bilinear) pairing defined by $\left\langle u_{i}, u_{j}\right\rangle=\delta_{i j}$, for all $i, j \in \bigcup B_{n}$.

Friedrichs' Theorem ([J. p. 170]). An element $a \in \overline{T V}$ is a Lie element if and only if $\theta a=1 \otimes a+a \otimes 1$; or equivalently, an element $w_{n} \in W^{\otimes n}(1 \leq n)$ is a Lie element if and only if $\theta w_{n}=1 \otimes w_{n}+w_{n} \otimes 1$.

Friedrichs' Theorem ([R, p. 214]). An element $a \in \overline{T V}$ is a Lie element if and only if $\langle a, b\rangle=0$, for all $b \in \overline{T V} \bullet \overline{T V}$. Equivalently, an element $w_{n} \in$ $V^{\otimes n}(1 \leq n)$ is a Lie element if and only if $\left\langle w_{n}, b_{n}\right\rangle=0$, for all $b_{n} \in V^{\otimes i} \bullet V^{\otimes j}$, whenever $i+j=n, 1 \leq i, j$.

As mentioned above, this second version of Friedrichs' theorem gives rise to the following dual formulation.

Dual Friedrichs' Theorem ([G, p. 458]). An element $f \in T c V$, with $f^{1}=0$ (that is, $\pi(f)=0)$, is a dual Lie element if and only if for each $2 \leq n, f^{n} \in$ $\overline{T V} \bullet \overline{T V}$.

Now we recall the Dynkin-Specht-Wever theorem, and then formulate and prove the "dual Dynkin-Specht-Wever theorem". For $1 \leq n$, let $\left[v_{1}, \ldots, v_{n}\right] \in$ $V^{\otimes n}$ denote the standard $n$-fold bracket defined inductively by $[v]=v$, and for $2 \leq n$

$$
\left[v_{1}, \ldots, v_{n}\right]=v_{1} \otimes\left[v_{2}, \ldots, v_{n}\right]-\left[v_{2}, \ldots, v_{n}\right] \otimes v_{1},
$$

for all $v, v_{1}, \ldots, v_{n} \in V$. As mentioned above, these elements span the space of Lie elements. One defines, on all homogeneous elements of degree $1 \leq n$, a linear map

$$
\gamma_{n}: V^{\otimes n} \rightarrow V^{\otimes n}, \quad v_{1} \cdots v_{n} \mapsto\left[v_{1}, \ldots, v_{n}\right],
$$

and therefore a linear map

$$
\gamma: \overline{T V} \rightarrow \overline{T V}, \quad \sum w_{n} \mapsto \sum \gamma_{n} w_{n},
$$

where $w_{n} \in V^{\otimes n}$. We can now state the following. 
Theorem (Dynkin-Specht-Wever [J, p. 169]). An element $a \in \overline{T V}$ is a Lie element if and only if $\gamma a=D a$; or equivalently, an element $w_{n} \in V^{\otimes n}(1 \leq n)$ is a Lie element if and only if $\gamma_{n} w_{n}=n w_{n}$.

We introduce the linear map (which will turn out to be analogous to $\gamma$ )

$$
\Gamma: T c V \rightarrow T c V, \quad f \mapsto(D-\rho) f .
$$

As far as we know, the following theorem has not previously appeared in the literature and will be proved in Theorem 8 .

Theorem 6 (Dual Dynkin-Specht-Wever). Let $f \in T c V$. Then $f$ is a dual Lie element if and only if $\Gamma f=D f$.

We remark that the condition $\Gamma f=D f$ in Theorem 6 is equivalent to requiring that $\rho f=0$. We choose to use the above form since it resembles the analogous condition of the Dynkin-Specht-Wever theorem.

Just as the Dynkin map, $\gamma$, has its image in (actually, equal to) the space of Lie elements, the next proposition, together with the implication (iii) implies (i) of Theorem 8 below, shows that our map, $\Gamma$, has its image in the space of dual Lie elements. Equivalently, Theorem 8 and the above remark show that the space of dual Lie elements is contained in (actually, equal to) the kernel of $\rho$.

Proposition 7. If $f \in T c V$, then $\Gamma f \in \overline{T c V} \bullet \overline{T c V}$.

Proof. Let $f=\sum_{0 \leq n} f^{n} \in T c V$. Then using (2) we find that

$$
\Gamma f=(D-\rho) f=\sum_{0 \leq n}\left(n f^{n}-\rho_{n} f^{n}\right)=\sum_{0 \leq n} \sum_{(f)} \sum_{i+j=n ; 1 \leq j} \rho_{i} f_{(1)}^{i} \bullet f_{(2)}^{j} .
$$

Since $\rho_{0}=0$, this is an element of $\overline{T c V} \bullet \overline{T c V}$.

Our map $\Gamma$ will now be used in order to give new and elementary proofs of both the dual Friedrichs' and dual Dynkin-Specht-Wever theorems. We note that in the following, the dual Friedrichs' theorem is the equivalence of (i) with (iii) and the dual Dynkin-Specht-Wever theorem is the equivalence of (i) with (ii).

Theorem 8. For $f \in T c V$, the following are equivalent:

(i) $f \in J$ ( $f$ is a dual Lie element).

(ii) $\Gamma f=D f$.

(iii) $f \in \overline{T c V} \bullet \overline{T c V} \oplus F$.

Proof. (i) implies (ii): To do this, we will show that $\rho f=0$ for all $f \in J$. By the u.m.p. of the cofree Lie coalgebra $L c V$, let $\bar{\pi}$ be the canonical Lie coalgebra map $\mathscr{L}_{c}(T c V) \rightarrow L c V$ induced by $\pi$, and let $f \in J$. An easy check shows that $J=\operatorname{ker} \bar{\pi}$. Since $\bar{\pi} f=0$, we have, by formula 10 and Lemma 10 of [G], for all $2 \leq n$,

$$
\begin{aligned}
0 & =\left(\bigotimes^{n} \pi\right)\left(\bigotimes^{n-2} 1 \otimes(1-\tau) \Delta\right) \cdots(1 \otimes(1-\tau) \Delta)(1-\tau) \Delta(f) \\
& =\left(\bigotimes^{n} \pi\right)\left(\bigotimes^{n-2} 1 \otimes(1-\tau) \Delta\right) \cdots(1 \otimes(1-\tau) \Delta)(1-\tau) \Delta\left(f^{n}\right) \\
& =\rho_{n} f^{n}
\end{aligned}
$$

Since $J \subseteq \operatorname{ker} \pi, f^{1}=0$, and we have $\rho f=0$; thus, (ii) holds. 
(ii) implies (iii): Using (ii) and Proposition 7, $D f \in \overline{T c V} \bullet \overline{T c V}$; therefore, (iii) holds.

(iii) implies (i): By Lemma $5, \overline{T c V} \bullet \overline{T c V}$ is a Lie coideal. Since every element of this Lie coideal has degree 1-component equaling 0 , this Lie coideal is contained in $\operatorname{ker} \pi$; hence, so is the Lie coideal $\overline{T c V} \bullet \overline{T c V} \oplus F$. This last Lie coideal is therefore contained in $J$ (being the largest such). Thus, (i) holds.

\section{ACKNOWLEDGMENT}

We would like to acknowledge that partial motivation for this work was due to the paper by Wigner [W].

\section{REFERENCES}

[A] M. Andre, Hopf algebras with divided powers, J. Algebra 18 (1971), 19-50.

[B-L] R. E. Block and P. Leroux, Generalized dual coalgebras of algebras, with applications to cofree coalgebras, J. Pure Appl. Algebra 36 (1985), 15-21.

[B] R. E. Block, Commutative Hopf algebras, Lie coalgebras, and divided powers, J. Algebra 96 (1985), 275-306.

[G] G. Griffing, The dual Friedrichs' theorem, J. Algebra 132 (1990), 446-460.

[J] N. Jacobson, Lie algebras, Dover, New York, 1979.

[M] W. Michaelis, Lie coalgebras, Adv. in Math. 38 (1980), 1-54.

[M1] _ The dual Poincare-Birkhoff-Witt theorem, Adv. in Math. 57 (1985), 159-161.

[M2] _ The primitives of the continuous linear dual of a Hopf algebra as the dual Lie algebra of a Lie coalgebra, Lie Algebras and Related Topics (Madison, WI, 1988), Contemp. Math., vol. 110, Amer. Math. Soc., Providence, RI, 1990, pp. 125-176.

[R] R. Ree, Lie elements and an algebra associated with shuffles, Ann. of Math. (2) 68 (1958), 210-220.

[S] M. E. Sweedler, Hopf algebras, Benjamin, New York, 1969.

[W] D. Wigner, An identity in the free Lie algebra, Proc. A.mer. Math. Soc. 106 (1989), 639-640.

Department of Mathematics, California State University, San Bernardino, CaliforNIA 92407

E-mail address: ggriffin@riley.csusb.edu 